\title{
GPDs and Fitting Procedures for DVCS
}

\author{
K. Kumerički \\ Physics Department, University of Zagreb, Bijenička c. 32, 10000 Zagreb, Croatia \\ kkumer@phy.hr \\ D. Mueller \\ Department of Physics, University of Cape Town, ZA-7701 Rondebosch, South Africa \\ dieter.mueller@tp2.rub.de
}

Published 29 February 2016

\begin{abstract}
We report on some approaches to extraction of generalized parton distributions (GPDs) from deeply virtual Compton scattering (DVCS) data.

Keywords: Generalized parton distributions, deeply virtual Compton scattering, neural networks.
\end{abstract}

PACS numbers: 13.60.-r, 13.60.Fz, 24.85.+p

\section{Introduction}

One of the prominent goals of hadron physics today is to determine the so called generalized parton distributions (GPDs) which are functions known to provide a detailed description of the nucleon in terms of partonic degrees of freedom ${ }^{1-3}$ (see also extensive reviews in Refs. [4] and [5]). The knowledge of GPDs would essentially give us the three-dimensional distribution of quarks and gluons inside the nucleon 6 and would also provide information about how the spin of the proton is built from the angular momenta of partons. ${ }^{7}$

Since GPDs are non-perturbative QCD quantities, they are not calculable from first principles, except for their first few moments that are measurable in QCD lattice simulations, see e.g. the review in Ref. [8]. Factorization theorems allow access to GPDs via flexible model building and fitting to experimental measurements of relevant observables, see recent reviews in Refs. [9] and [10].

In this note we report on and compare several methods of GPD extraction with emphasis on local fits and neural networks approach.

This is an Open Access article published by World Scientific Publishing Company. It is distributed under the terms of the Creative Commons Attribution 3.0 (CC-BY) License. Further distribution of this work is permitted, provided the original work is properly cited. 


\section{Methods and Results}

The most popular method for accessing GPDs involves the comparison of model predictions with data on cross-sections and asymmetries of deeply virtual Compton scattering (DVCS), $\gamma^{*} p \rightarrow \gamma p$, which is accessible in the exclusive electroproduction of a photon, see e.g. Refs. $[11,12]$ and comments in Refs. $[9,13]$. Note that via the optical theorem the deeply virtual Compton scattering in forward kinematics, $\gamma^{*} p \rightarrow$ $\gamma^{*} p$, is related to inclusive deep inelastic scattering (DIS). Correspondingly, just like in DIS the virtual photon probes the partonic structure of a hadron. However, in DVCS this structure is probed by two photons, making the probe more powerful, but also more difficult to use. The DVCS amplitude can be parameterized in terms of Compton form factors (CFFs), which are given by the convolution of GPDs with the Wilson coefficient functions from the operator product expansion of two electromagnetic currents. E.g. for the best known $\mathrm{CFF} \mathcal{H}$ and GPD $H$ we have to leading order (LO) accuracy

$$
\mathcal{H}\left(x_{B}, t, \mathcal{Q}^{2}\right) \stackrel{\mathrm{LO}}{=} \int_{-1}^{1} d x\left[\frac{1}{\xi-x-i \epsilon}-\frac{1}{\xi+x-i \epsilon}\right] H\left(x, \xi, t, \mathcal{Q}^{2}\right),
$$

where $\xi=x_{B} /\left(2-x_{B}\right), x_{B}$ is Bjorken's scaling variable, $t$ is the square of the momentum transfer, and $q^{2}=-\mathcal{Q}^{2}$ is the photon virtuality. It is often stressed that this convolution formula makes it difficult to extract the $(x, \xi)$-shape of the GPD. As we will see below, for the imaginary part the corresponding DIS formula, expressing the structure function $F_{1}$ in terms of quark parton distribution functions (PDFs) $q\left(x, Q^{2}\right)$, is essentially the same, where the corresponding PDFs are known to relatively high accuracy. Nevertheless, the dimensionality of the GPD domain space is a serious obstacle in the search for the shape of GPD functions, which might be overcome to some extent by a combined use of experimental and lattice data. Of the four variables on which $\operatorname{GPD} H\left(x, \xi, t, \mathcal{Q}^{2}\right)$ depends, only the dependence on the photon virtuality $\mathcal{Q}^{2}$ is predicted by perturbative QCD evolution equations, which is also determined by the $(x, \xi)$-shape of the GPD at the input scale. Knowledge of dependence on the other three kinematic variables, longitudinal momentum fraction $x$, skewness $\xi$, or momentum transfer squared $t$ is poor. The general properties of GPDs, such as the relations to PDFs, elastic form factors, and generalized form factors, constrain GPDs only on borders of its domain space or by sum rules, while GPDs in the "bulk" have to be determined by fits to independent experiments or possibly by assuming some "holographic" principle, ${ }^{13}$ which in turn might characterize a certain class of GPD models.

In the lack of truly reliable GPD models (which is the present state of affairs), and faced with the multi-dimensional GPD domain space, currently available experimental data are not sufficient to facilitate extraction of most GPDs. One possible strategy to approach this problem is to determine first just the CFFs. These quantities depend on one kinematic variable less than GPDs, see (1), which makes them more constrained by measurements. Also, the DVCS coefficient functions at leading 
order of perturbation theory ("handbag" approach) are such that in this order the imaginary part of CFFs determine the GPDs on the so-called cross-over line $x=\xi$,

$$
\frac{1}{\pi} \Im m \mathcal{H}\left(x_{B}=\frac{2 \xi}{1+\xi}, t, \mathcal{Q}^{2}\right) \stackrel{\mathrm{LO}}{=} H\left(x=\xi, \xi, t, \mathcal{Q}^{2}\right)-H\left(x=-\xi, \xi, t, \mathcal{Q}^{2}\right) .
$$

Hence, knowledge of CFFs gives us also some direct information about GPDs. This can be particularly well exploited if one uses dispersion relations to relate real and imaginary part of CFFs - then the whole leading order (and leading twist) phenomenology is determined by GPDs on the cross-over line and by a dispersion relation subtraction constant that is related to the so-called $D$-term. ${ }^{14}$ Such an approach was studied in Ref. [15] and was utilized for the valence quark sector in Ref. [16] to perform a global GPD model fit, denoted as KMM12, that describes available DVCS data from both collider and fixed target experiments reasonably well. $^{\mathrm{a}}$

Relying on the scaling hypothesis, which might be justified for the existing data from fixed target experiments, even modelling the GPDs on the cross-over line (two-dimensional $x_{B}$ - $t$ domain space) and subtraction constant (one-dimensional $t$ domain space) necessarily entails some model bias. In the analogous task of determination of PDFs, this problem is not so pronounced because domain space is one-dimensional (just $x_{B}$ ), and large number of experimental data points is available. But here situation is much more problematic, and looking ahead and thinking about actual modelling of complete GPD $F(x, \xi, t)$ functions, we should better be able to assess and control the amount of model bias we introduce.

One useful method, which is not sensitive to model bias, is performing local fits to the data, where one extracts CFF values at a given kinematic point at which possibly several different observables are measured. This is particularly interesting in the case of DVCS measurements by HERMES collaboration, where indeed an almost complete set of observables is measured in 12 kinematic bins. ${ }^{17-20}$ In HERMES kinematics, many measured asymmetries in $e p \rightarrow e p \gamma$ scattering are dominated by the interference of DVCS (final photon emitted from proton) and Bethe-Heitler scattering (photon emitted from lepton) amplitudes, and are thus almost linear in CFFs. This leads to a well-defined mapping from asymmetries to CFFs and quite a direct propagation of uncertainties. Study in Ref. [16] shows that few CFFs can be clearly and unambiguously extracted, confirming previous similar findings. ${ }^{21,22}$ CFFs extracted by such mapping method are depicted on Fig. 1, where they are compared to CFFs extracted by standard least-squares local fits. As expected, the imaginary part of $\mathrm{CFF} \mathcal{H}$ (determined by GPD $H$, cf. (1)) is the most reliably extracted quantity, thanks to its kinematically unsuppressed appearance in most observables.

Such local fitting exercises are convenient tools to assess general consistency of the whole theoretical and experimental GPD physics framework, thanks to the

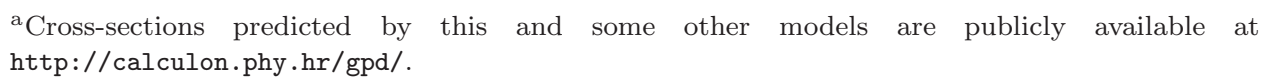



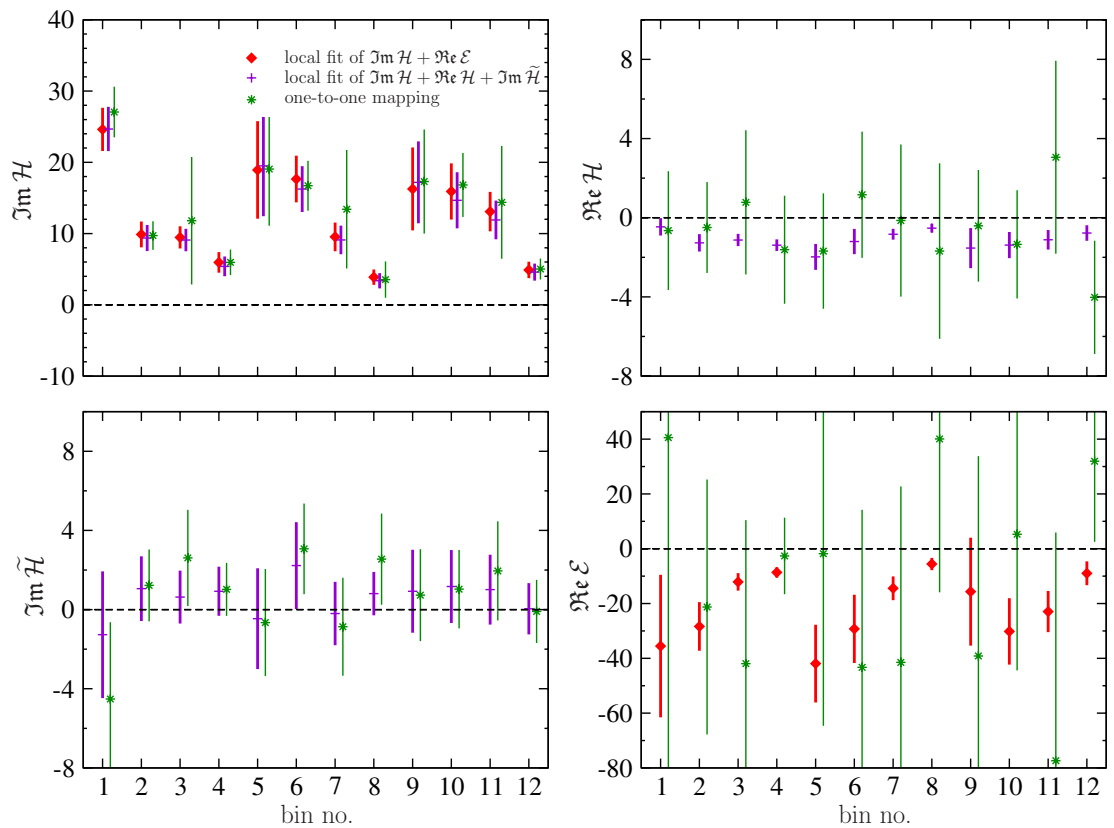

Fig. 1. Several local fits to HERMES data in 12 kinematic bins. Figure is taken from Ref. [16], where also specification of kinematic bins can be found. Green stars are obtained by method of mapping observables to all eight leading imaginary and real parts of CFFs, while red squares and blue plusses are least-squares fits to a small number of dominant CFFs.

absence of modelling bias. However, they are of limited use and should be considered only as an intermediate step in the whole endeavour. Another method, which sits nicely between model-independent local and model-dependent global fits is the method of neural networks. This method promises in some sense to be the best of both worlds: It is a global method, that can give us shape of the GPDs or CFFs away from measured points (with uncertainty increasing away from these points in a well-defined way), while at the same time being free of modelling bias, if one carefully applies some method of overfitting avoidance, such as some form of crossvalidation. A first study, showing the viability of neural network approach on the limited set of beam spin asymmetry and beam charge asymmetry HERMES data, is described in Ref. [23] (see also toy example in Ref. [24]).

\section{Conclusion}

As described, several different fitting approaches have been tried in attempt to extract the shapes of dominant structure functions (CFFs) and their associated partonic quantities (GPDs), which contain also information on the transversal partonic structure of the proton in the kinematics of HERMES experiment. There is a clear agreement of the results from the different methods, showing consistency of the whole framework. These studies give a clear idea of GPD information that can 
be accessed from presently available data, and can be used for guidance in finding physically motivated GPD models, flexible enough to describe these data. For the future it is expected that JLAB experiments will provide new high quality data for an extended set of observables, which might allow in a global DVCS analysis to remove, as it is already possible for HERMES data, assumptions and hypotheses, while a planned COMPASS-II experiment will help to fill the kinematical gap between collider and fixed target data from HERA. To obtain a reliable determination of uncertainties of extracted GPDs/CFFs, the precise information about correlations of uncertainties of experimental measurements would be very welcome, like it has become standard in DIS experiments.

\section{Acknowledgments}

This work has been supported in part by the Croatian Science Foundation under the project number 8799 .

\section{References}

1. D. Müller, D. Robaschik, B. Geyer, F. M. Dittes and J. Hořejší, Fortschr. Phys. 42, 101 (1994), [hep-ph/9812448].

2. A. V. Radyushkin, Phys. Lett. B380, 417 (1996), [hep-ph/9604317].

3. X.-D. Ji, Phys. Rev. D55, 7114 (1997), [hep-ph/9609381].

4. M. Diehl, Phys. Rept. 388, 41 (2003), [hep-ph/0307382].

5. A. V. Belitsky and A. V. Radyushkin, Phys. Rept. 418, 1 (2005), [hep-ph/0504030].

6. M. Burkardt, Phys. Rev. D62, 071503 (2000), [hep-ph/0005108], Erratumibid.D66:119903,2002.

7. X.-D. Ji, Phys. Rev. Lett. 78, 610 (1997), [hep-ph/9603249].

8. Ph. Hägler, Phys. Rept. 490, 49 (2010), [hep-lat/0912.5483].

9. D. Müller, Few Body Sys. 55, 317 (2014), [1405.2817].

10. M. Guidal, H. Moutarde and M. Vanderhaeghen, Rept.Prog.Phys. 76, 066202 (2013), [1303.6600].

11. M. Vanderhaeghen P. A. M. Guichon and M. Guidal, Phys. Rev. D60, 094017, [hep$\mathrm{ph} / 9905372]$.

12. P. Kroll, H. Moutarde and F. Sabatie, Eur. Phys. J. C73, 2278 (2013), [1210.6975].

13. K. Kumerički, D. Müller and K. Passek-Kumerički, Eur. Phys. J. C58, 193 (2008), [0805.0152].

14. M. V. Polyakov and C. Weiss Phys. Rev. D60, 114017 (1999), [hep-ph/9902451].

15. K. Kumerički and D. Müller, Nucl. Phys. B841, 1 (2010), [0904.0458].

16. K. Kumerički, D. Müller and M. Murray, Phys. Part. Nucl. 45, 723 (2014), [1301.1230].

17. HERMES, A. Airapetian et al., JHEP 06, 066 (2008), [0802.2499].

18. HERMES Collaboration, A. Airapetian et al., Phys. Lett. B704, 15 (2011), [1106.2990].

19. HERMES Collaboration, A. Airapetian et al., JHEP 1207, 032 (2012), [1203.6287].

20. HERMES Collaboration, A. Airapetian et al., JHEP 1210, 042 (2012), [1206.5683].

21. M. Guidal, Eur. Phys. J. A37, 319 (2008), [0807.2355].

22. M. Guidal and H. Moutarde, Eur. Phys. J. A42, 71 (2009), [0905.1220].

23. K. Kumerički, D. Müller and A. Schäfer, JHEP 07, 073 (2011), [1106.2808].

24. K. Kumerički, D. Müller and A. Schäfer, Nucl.Phys.Proc.Suppl. 222-224, 199 (2012), [1112.1958]. 